\title{
Aberrant Patterning of Neuromuscular Synapses in Choline Acetyltransferase-Deficient Mice
}

\author{
Eugene P. Brandon, ${ }^{1 \star}$ Weichun Lin, ${ }^{2 \star}$ Kevin A. D’Amour, ${ }^{1}$ Donald P. Pizzo, ${ }^{3}$ Bertha Dominguez, ${ }^{2}$ Yoshie Sugiura, ${ }^{4}$ \\ Silke Thode, ${ }^{1}$ Chien-Ping Ko, ${ }^{4}$ Leon J. Thal, ${ }^{3}$ Fred H. Gage, ${ }^{1}$ and Kuo-Fen Lee ${ }^{2}$ \\ ${ }^{1}$ Laboratory of Genetics and ${ }^{2}$ Peptide Biology Laboratories, The Salk Institute for Biological Studies, La Jolla, California 92037, ${ }^{3}$ Department of \\ Neurosciences, School of Medicine, University of California at San Diego, La Jolla, California 92037, and ${ }^{4}$ Neurobiology Section, Department of Biological \\ Sciences, University of Southern California, Los Angeles, California 90089
}

In this study we examined the developmental roles of acetylcholine (ACh) by establishing and analyzing mice lacking choline acetyltransferase (ChAT), the biosynthetic enzyme for ACh. As predicted, ChAT-deficient embryos lack both spontaneous and nerve-evoked postsynaptic potentials in muscle and die at birth. In mutant embryos, abnormally increased nerve branching occurs on contact with muscle, and hyperinnervation continues throughout subsequent prenatal development. Postsynaptically, ACh receptor clusters are markedly increased in number and occupy a broader muscle territory in the mutants. Concomitantly, the mutants have significantly more motor neurons than normal. At an ultrastructural level, nerve terminals are smaller in mutant neuromuscular junctions, and they make fewer synaptic contacts to the postsynaptic muscle membrane, although all of the typical synaptic components are present in the mutant. These results indicate that ChAT is uniquely essential for the patterning and formation of mammalian neuromuscular synapses.

Key words: choline acetyltransferase; acetylcholine; neural development; mice; neuromuscular; gene knock-out

\section{Introduction}

The study of acetylcholine (ACh) and cholinergic functions has a long and rich history (for review, see Karczmar, 1996). Numerous studies have focused on the role of ACh in the development of spinal motor neurons and their peripheral synapses, neuromuscular junctions (NMJs). Both the patterning and formation of NMJs require coordinated interactions between the nerve terminals, Schwann cells, and muscle cells (Burden, 1998; Sanes and Lichtman, 1999). NMJs do not develop at random locations in muscles; instead each is assembled in a narrow central region of its muscle fiber, with many NMJs in a row forming an "end plate band" across fibers. It has recently come to light that the central region of the muscle fiber is endowed with intrinsic signals for the initiation of synaptogenesis. By mouse embryonic day 14.5 (E14.5), ACh receptors (AChRs) have begun to form clusters in the central band of muscle via a nerve-independent mechanism (Lin et al., 2001; Yang et al., 2001). Many of these early AChR clusters are not apposed by nerve terminals. Subsequently, the nerve provides both positive and negative signals that promote additional development of the NMJ. These neural signals regulate the width of the end plate band, the differentiation and stabilization of AChR clusters, and the dispersion of AChR clusters not stabilized by the nerve (Davis et al., 2001; Ferns and Carbonetto,

\footnotetext{
Received May 17, 2002; revised 0ct. 21, 2002; accepted 0ct. 30, 2002.

This work was supported by grants from the National Institutes of Health/National Institute on Aging, the Muscular Dystrophy Association, and the John Douglas French Alzheimer's Foundation. K.F.L. is a Pew Scholar. We thank Kevin Gobeske, Nushin Sherkat, James Henry, Lynne Moore, Bobbi Miller, Linda Kitabayashi, Steve Forbes, Andrew Chen, Samir Koirala, and Yelena Dayn for excellent technical assistance, and Mu-Ming Poo, Steve Heinemann, Rebecca Tuttle, Rebecca Cole, and Mary L. Gage for helpful comments on this manuscript.

*E.P.B. and W.L. contributed equally to this study.

Correspondence should be addressed to Dr. Fred H. Gage, Laboratory of Genetics, The Salk Institute for Biological Studies, 10010 North Torrey Pines Road, La Jolla, CA 92037. E-mail: gage@salk.edu.

E. P. Brandon's present address: Ceregene, Inc., 9381 Judicial Drive, San Diego, CA 92121.

Copyright $\odot 2003$ Society for Neuroscience $\quad 0270-6474 / 03 / 230539-11 \$ 15.00 / 0$
}

2001; Lin et al., 2001; Yang et al., 2001). From these recent results, two important questions arise. First, what roles do these nerveindependent $\mathrm{AChR}$ clusters have in the patterning and formation of synapses? Second, is the region outside of the central band of developing muscle capable of forming synapses? We postulate that $\mathrm{ACh}$ released from the incoming motor nerve acts through these nerve-independent AChR clusters to make the central band of muscle particularly permissive for both the cessation of nerve growth and the induction of synapse formation. Alternatively, or in addition, the muscle region outside the central band may contain the necessary machinery for synapse formation but becomes nonreceptive for nerve terminals as a result of ACh activation of the nerve-independent AChRs in the central band of muscle. We hypothesize that through one or both of these mechanisms, ACh contributes to the centralization of NMJs (Lin et al., 2001).

Previous studies to probe the role of ACh in NMJ development have relied primarily on anticholinergic or activity-blocking agents. Several investigators have used the application of AChR antagonists such as $d$-turbocurare (dTC) to chicks in ovo (Burden, 1977; Pittman and Oppenheim, 1978; Srihari and Vrbova, 1978; Dahm and Landmesser, 1988, 1991; Oppenheim et al., 1989, 2000; Hory-Lee and Frank, 1995; Usiak and Landmesser, 1999), an approach that has proved to be very informative. However, the analogous in utero pharmacological interventions in rodents have been of limited use because at stages before nerve-muscle contact, the manipulations have dire effects on the pregnant mother and/or developing fetuses (Braithwaite and Harris, 1979; Houenou et al., 1990). Furthermore, some results between chicks and rodents have been discordant. For example, although the regulation of motor nerve branching by ACh was observed in chicks, it was not seen in mice (Houenou et al., 1990). This difference may be attributable to the technical difficulty of the application of dTC before the arrival of nerves at the muscle in mouse embryos. 
To reconcile and extend previous results and to directly examine the physiological role of ACh in the patterning and formation of neuromuscular synapses in mammals, we used gene targeting to nullify the Chat gene in mice and eliminate choline acetyltransferase (ChAT; acetyl-coenzyme A:choline O-acetyltransferase; EC 2.3.1.6), the biosynthetic enzyme for ACh. Our results confirm and extend many of the previous findings from chicks. In particular, Chat null mutants exhibit muscle hyperinnervation and increased motor neuron survival. The mutants show a concomitant broadening of the muscular territory occupied by synapses. By late gestation, the synapses in mutants are abnormal morphologically. The results indicate that $\mathrm{ACh}$ is required to regulate axonal growth and to determine the location of synapses in the muscle.

Portions of this study have been published previously in abstract form (Brandon et al., 2000).

\section{Materials and Methods}

Generation of mutant mice. Although the genomic structure of mouse Chat has not been described, it is known that in rats and humans, ChAT is encoded by a large gene that includes 14 coding exons in the $3^{\prime}$ region that are used in all transcripts. In contrast, the $5^{\prime}$ region of the gene is complicated, with alternative splicing, multiple transcription and translation start sites, and the gene encoding the vesicular ACh transporter embedded in the first intron (Hahn et al., 1992; Ohno et al., 2001). To avoid this $5^{\prime}$ region, the catalytic portion of the protein was targeted for deletion. Site-directed mutagenesis of Drosophila ChAT has shown that conserved histidines (H302 and H335 in mice) are critical for enzymatic activity (Carbini and Hersh, 1993). Point mutations in this same region of the human gene reduce protein expression and lower the efficiency of ACh synthesis (Ohno et al., 2001). We found the genomic structure of this region of mouse Chat to be homologous to those described for rat and human genes. As shown in Figure $1 A$, the targeting vector was designed to replace exons 11,12 , and 13 [using the recent nomenclature used to describe human CHAT (Ohno et al., 2001)] with a neomycin resistance cassette expressed in the opposite orientation from Chat. The targeting vector was linearized with NotI and electroporated into J1 embryonic stem (ES) cells as described previously (Lee et al., 1992). After

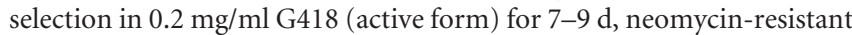
clones were isolated and screened for the presence of the disrupted Chat allele by Southern blot analysis. Positive ES clones were injected into C57BL/6 blastocysts to generate chimeric mice. PCR of tail DNA with a phosphoglycerate kinase-neomycin (pgk-neo)-specific oligonucleotide, a Chat exon 13 oligonucleotide, and a shared Chat intronic oligonucleotide was used for genotyping. The use of animals is in compliance with the guidelines of the Animal Care and Use Committee of the Salk Institute.

ChAT assay. Tissue was homogenized by sonication in $100 \mu \mathrm{l}$ of a solution containing $0.1 \%$ Triton X-100 and $0.87 \mathrm{~mm}$ EDTA, $\mathrm{pH} 7.0$, and was not further diluted because of the low activity present at this stage. ChAT activity was assayed in triplicate by the incorporation of ${ }^{14} \mathrm{C}$ acetyl-coenzyme A into ${ }^{14} \mathrm{C}$-acetylcholine as described previously and is expressed as nanomoles of ACh synthesized per hour per milligram of protein (Pizzo et al., 1999).

Electrophysiology. Intracellular sharp-electrode recording was performed blind to genotype on phrenic nerve/diaphragm preparations from E17.5 embryos. Tissue was dissected in oxygenated normal mouse Ringer's (NMR) solution (in mM): $135 \mathrm{NaCl}, 5 \mathrm{KCl}, 15 \mathrm{NaHCO}_{3}, 1$ $\mathrm{Na}_{2} \mathrm{HPO}_{4}, 1 \mathrm{MgSO}_{4}, 2.5$ Ca gluconate, and 11 glucose, $\mathrm{pH}$ 7.4, pinned to Sylgard-coated dishes, and continuously perfused with oxygenated NMR. Glass microelectrodes filled with $3 \mathrm{M} \mathrm{KCl}$ were used to record spontaneous miniature end plate potentials (mepps) at $22-24^{\circ} \mathrm{C}$ for $\sim 5$ min. End plate potentials (epps) were evoked by suprathreshold stimulation of the phrenic nerve via suction electrode and were recorded in NMR containing $10 \mathrm{~mm}$ Ca gluconate and 5-12 mM dTC (to prevent muscle contractions). Data were collected and analyzed using pClamp (version 8.0; Axon Instruments, Foster City, CA) and Minianalysis (Synaptosoft, Decatur, GA).

Motor neuron counts and stereology. Spinal columns were isolated from embryos ( $n=5$ wild-type embryos and 7 mutants) fixed in $4 \%$ paraformaldehyde (PFA), equilibrated with 30\% sucrose, and transversesectioned at $14 \mu \mathrm{m}$ thickness. Sections were stained with $2 \%$ thionine solution. Motor neurons were counted blind to genotype, bilaterally, based on morphology and location in every 16th section, and values were multiplied by the number of sections to generate total estimates. Only cells with a clear nucleus and nucleolus were counted. A two-tailed $t$ test was used to determine statistical significance. Despite the kyphosis of the mutants, the distance of spinal segments from C2 to T6 did not differ between genotypes (data not shown). Stereological measurements of motor neuron size were performed on all of the counted cells on every 64th section, and the average motor neuron size for each spinal segment examined was determined for each embryo. In total, 1986 profiles were measured in 12 embryos. ANOVA was used to determine statistical significance.

Immunocytochemistry. For ChAT immunohistochemistry, embryos were fixed in 4\% PFA, equilibrated with $30 \%$ sucrose, and coronally sectioned at $25 \mu \mathrm{m}$ thickness. Sections were dried on glass slides, rinsed (three times with $0.1 \mathrm{M}$ TBS), incubated for $45 \mathrm{~min}$ in $0.6 \% \mathrm{H}_{2} \mathrm{O}_{2}$ in $0.1 \mathrm{M}$ TBS, rinsed again, incubated for $1 \mathrm{hr}$ in blocking buffer ( $0.1 \mathrm{~m} \mathrm{TBS,} \mathrm{5 \%}$ donkey serum, and $0.1 \%$ Triton X-100), and then incubated overnight at $4^{\circ} \mathrm{C}$ in primary goat antibody against ChAT $(1: 100$; Chemicon, Temecula, CA) in blocking buffer. The slides were rinsed, incubated in biotinylated donkey anti-goat IgG (1:250; Jackson ImmunoResearch, West Grove, PA) in blocking buffer for $3 \mathrm{hr}$, rinsed, incubated in ABCElite reagent (Vector Laboratories, Burlingame, CA) for $1 \mathrm{hr}$, rinsed, and developed in DAB. For whole-mount analyses, embryos were fixed in $2 \%$ PFA in $0.1 \mathrm{M}$ phosphate buffer, $\mathrm{pH} 7.3$, at $4^{\circ} \mathrm{C}$ overnight. Diaphragm and intercostal muscles were dissected, rinsed briefly with PBS, pH 7.3, incubated in $0.1 \mathrm{~m}$ glycine in PBS for $1 \mathrm{hr}$, and rinsed briefly with PBS and then with $0.5 \%$ Triton X-100 in PBS. The muscles were blocked in dilution buffer ( $150 \mathrm{~mm} \mathrm{NaCl}, 0.01 \mathrm{~m}$ phosphate buffer, $3 \%$ BSA, 5\% goat serum, and $0.01 \%$ thimerosal) overnight at $4^{\circ} \mathrm{C}$ and then incubated with primary rabbit antibodies against neurofilament 150 (1:1000; Chemicon), synaptophysin (1:1000; a gift from P. DeCamilli, Yale University, New Haven, CT), or $S 100 \beta$ (1:500; a Schwann cell marker) in dilution buffer overnight at $4^{\circ} \mathrm{C}$. After washing three times for $1 \mathrm{hr}$ each in $0.5 \%$ Triton $\mathrm{X}-100$ in PBS, the muscles were incubated in fluorescein isothiocyanateconjugated goat anti-rabbit IgG (1:600; Cappel, Cochranville, PA) and Texas Red-conjugated $\alpha$-bungarotoxin $\left(10^{-8} \mathrm{M}\right.$; Molecular Probes, Eugene, OR) overnight at $4^{\circ} \mathrm{C}$. The muscles were then washed three times for $1 \mathrm{hr}$ each with $0.5 \%$ Triton X-100 in PBS, washed once with PBS, and flat-mounted in $90 \%$ glycerol, polyvinyl alcohol, and $N$-propyl gallate. Images were collected with an Olympus Optical (Tokyo, Japan) confocal microscope. For quantitative analysis of AChR clusters, the numbers of AChR clusters in a matching $4.8 \times 10^{5} \mu \mathrm{m}^{2}$ area of the ventral-costal portion of diaphragm muscle from control $(n=3)$ and mutant $(n=3)$ embryos were counted. The data were collected in $100 \mu \mathrm{m}$ bins emanating from the medial edge of the muscle, perpendicular to the muscle fibers, and results were expressed as an average number of AChR clusters in each $100 \mu \mathrm{m}$ interval.

Acetylcholinesterase histochemistry. Tissues were dissected from embryos or postnatal animals fixed with $4 \%$ PFA, rinsed in TBS several times, and incubated in a solution of (in $\mathrm{mm}$ ): 0.2 ethopropazine, 4 acetylthiocholine iodine, 10 glycine, 2 cupric sulfate, and 65 sodium acetate solution, $\mathrm{pH} 5.5$, for $2-4 \mathrm{hr}$ at $37^{\circ} \mathrm{C}$. Staining for acetylcholinesterase (AChE) was developed by incubating the tissues for $2-5 \mathrm{~min}$ in sodium sulfide (1.25\%), pH 6.0. The tissues were then washed extensively with water, cleared with $50 \%$ glycerol in PBS, and mounted on a glass slide before imaging.

In situ hybridization. For whole-mount in situ hybridization, diaphragm muscles were fixed in $4 \% \mathrm{PFA}$ in $0.1 \mathrm{M}$ phosphate buffer at $4^{\circ} \mathrm{C}$ overnight. Digoxigenin-labeled AChR $\alpha$ cRNA probes were transcribed in vitro. Hybridization was performed at $70^{\circ} \mathrm{C}$ overnight in the hybridization buffer containing $50 \%$ formamide, $1.3 \times$ SSC, 5 mM EDTA, 50 $\mu \mathrm{g} / \mathrm{ml}$ yeast tRNA, $0.2 \%$ Tween $20,0.5 \%$ 3-[(3-cholamidopropyl) 



Figure 2. Synaptic transmission is absent in Chat mutant NMJ. A, Spontaneous mepps were observed in control $(+/-)$ diaphragm but not in the null mutant $(-/-)$ diaphragm. One mepp is expanded below. $B, 0$ treatment with potassium chloride, a drastic increase in mepp frequency was observed in the control $(+/-)$ but not in the mutant $(-I-)$. $C$, Nerve-evoked epps were readily detected in control $(+/-)$ but not in mutant $(-/-)$ muscle fibers. Averaged responses from 11 to 12 fibers per genotype are shown. $D$, The ACh agonist carbachol elicited synaptic responses in both control $(+/-)$ and mutant $(-/-)$ muscles, demonstrating that AChR clusters are functional in the mutants (arrows indicate time of carbachol application).

dimethylammonio]-1-propanesulfonate, and $100 \mu \mathrm{g} / \mathrm{ml}$ heparin. After hybridization, the samples were washed three times with TBS containing $1 \%$ Tween 20 for $1 \mathrm{hr}$ each, blocked with $5 \%$ goat serum in dilution buffer, and incubated with alkaline phosphatase conjugated antidigoxigenin (1:1000; Boehringer Mannheim, Indianapolis, IN) overnight

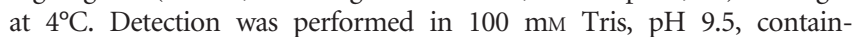
ing nitroblue-tetrazolium-chloride/5-bromo-4-chlor-indolyl-phosphate. To determine whether AChR transcript is found at synaptic sites, we performed in situ hybridization analysis on frozen sections of diaphragm muscles that had been processed for AChE staining (see above). Briefly, diaphragm muscle was stained for AChE, fixed in 4\% PFA, cryoprotected in

Figure 1. Generation of Chat mutant mice. A, Top, The genomic region of the Chat gene containing exons 11-14. Middle, The targeting vector, constructed by deleting a fragment of genomic DNA containing exons 11-13 and replacing it with a pgk-neo cassette (neo), contained $4.9 \mathrm{~kb}$ of $5^{\prime}$ and $2.1 \mathrm{~kb}$ of $3^{\prime}$ homologous DNA. Bottom, The resulting mutated Chat allele. $B$, $B a m H I . B, E S$ clones that underwent homologous recombination were identified by Southern blot analysis. DNA was digested with $B a m H I$ and hybridized with the probe depicted in $A$, which detects a $9.1 \mathrm{~kb}$ wild-type (WT) band and an $8.7 \mathrm{~kb}$ mutant (MUT) band. Lane 4 is a heterozygous clone. C, PCR analysis of embryos clearly identifies the different genotypes. D, ChAT activity (nanomoles per hour per milligram of protein) was determined in brain stem (bst), spinal cord

(sc), and septum (spt) samples collected from E18.5 embryos. Only background activity was detected in homozygous (open bars; - I-) Chat mutants. Samples from heterozygous (shaded bars; +/-) embryos contained approximately half of the control ( filled bars; +/+) ChAT activity. Error bars indicate SEM. E, Immunohistochemistry demonstrated that ChAT immunoreactivity is not detected in the nucleus basalis of mutant $(-/-)$ mice. Scale bar, $100 \mu \mathrm{m}$. F, An E16.5 Chat mutant (-/-) embryo compared with a control (+/+) littermate. 

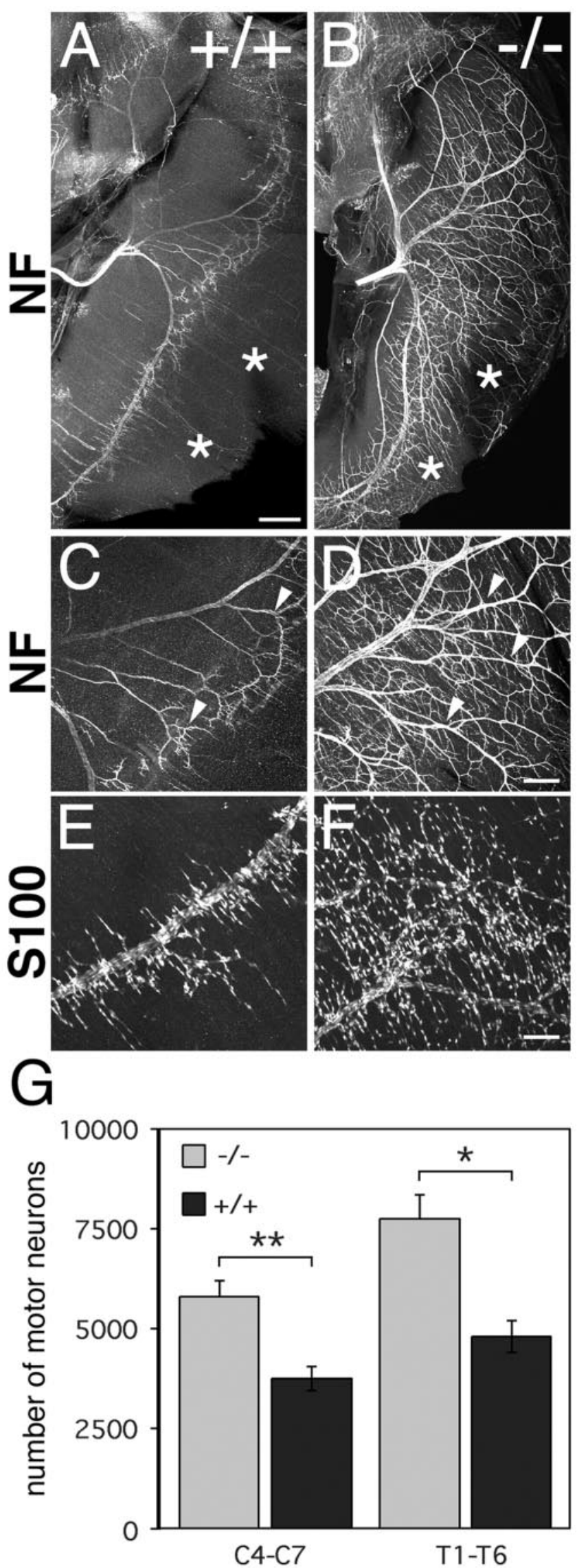

Figure 3. Hyperinnervation of Chat mutant muscle. E18.5 diaphragm muscles were collected from control $(A ;+/+)$ and mutant $(B ;-/-)$ embryos and immunostained with


Figure 4. Increased nerve branching on first contact with muscle. Diaphragm anlages were collected from control $(A ;+/+)$ and mutant $(B ;-/-)$ embryos at E12.5 and immunostained with anti-NF antibodies. The phrenic nerve in control embryos is tightly bundled, with little defasciculation. In contrast, the phrenic nerve in the mutants is highly branched. Primary $(p)$, secondary $(s)$, and tertiary $(t)$ branches of the phrenic nerve are shown. A similar phenomenon is seen with the innervation of the intercostal muscles: control $(C)$ nerve grows primarily in a single fascicle, whereas in the mutant $(D)$, highly branched axons emerge from the bundle. Increased nerve branching is pronounced in E13.5 mutant diaphragm muscles $(F)$ compared with controls (E). Scale bars: (in D) A-D, $100 \mu \mathrm{m}$; (in F) E, F, $500 \mu \mathrm{m}$.

$30 \%$ sucrose, and sectioned at $20 \mu \mathrm{m}$ thickness. Sections were hybridized with a ${ }^{33} \mathrm{P}$-labeled $\mathrm{AChR} \alpha$ riboprobe. Slides were dipped in liquid nuclear emulsion (type NTB2; Kodak, Rochester, NY) and exposed for 5 d. Finally, slides were photographically processed and counterstained with hematoxylin and eosin.

Electron microscopy. E17.5 pregnant females were killed by cervical dislocation, and the embryos were removed. After the tail was removed for PCR analysis, the remainder of the body was placed in a solution of $2 \%$ glutaraldehyde in $0.1 \mathrm{~m}$ phosphate buffer, $\mathrm{pH}$ 7.4. The diaphragm

$\leftarrow$

anti-NF antibodies. An effusion of nerve branching (arrowheads in ( and $D$ ) as well as extensive innervation of normally nonpermissive regions (asterisks) were observed in the diaphragm muscles of mutants. At higher-power magnification, detailed analysis indicates that axons not only leave, but also rejoin, nerve bundles in mutant embryos $(D)$. $5100 \beta$-immunoreactive Schwann cells are present in both control $(E)$ and mutant $(F)$ embryos. Scale bars: (in $A) A, B$, $500 \mu \mathrm{m}$; (in D) C, D, $200 \mu \mathrm{m}$; (in F) E, F, $50 \mu \mathrm{m}$. G, Motor neuron counts revealed that the number of motor neurons was significantly increased in the Chat mutant $(-/-)$ embryos in both cervical ( $\left(4-\mathrm{C} 7 ;{ }^{* *} p<0.005\right)$ and thoracic $\left(\mathrm{T} 1-\mathrm{T} 6 ;{ }^{*} p<0.01\right)$ spinal regions compared with wild-type control $(+/+)$ littermates. 

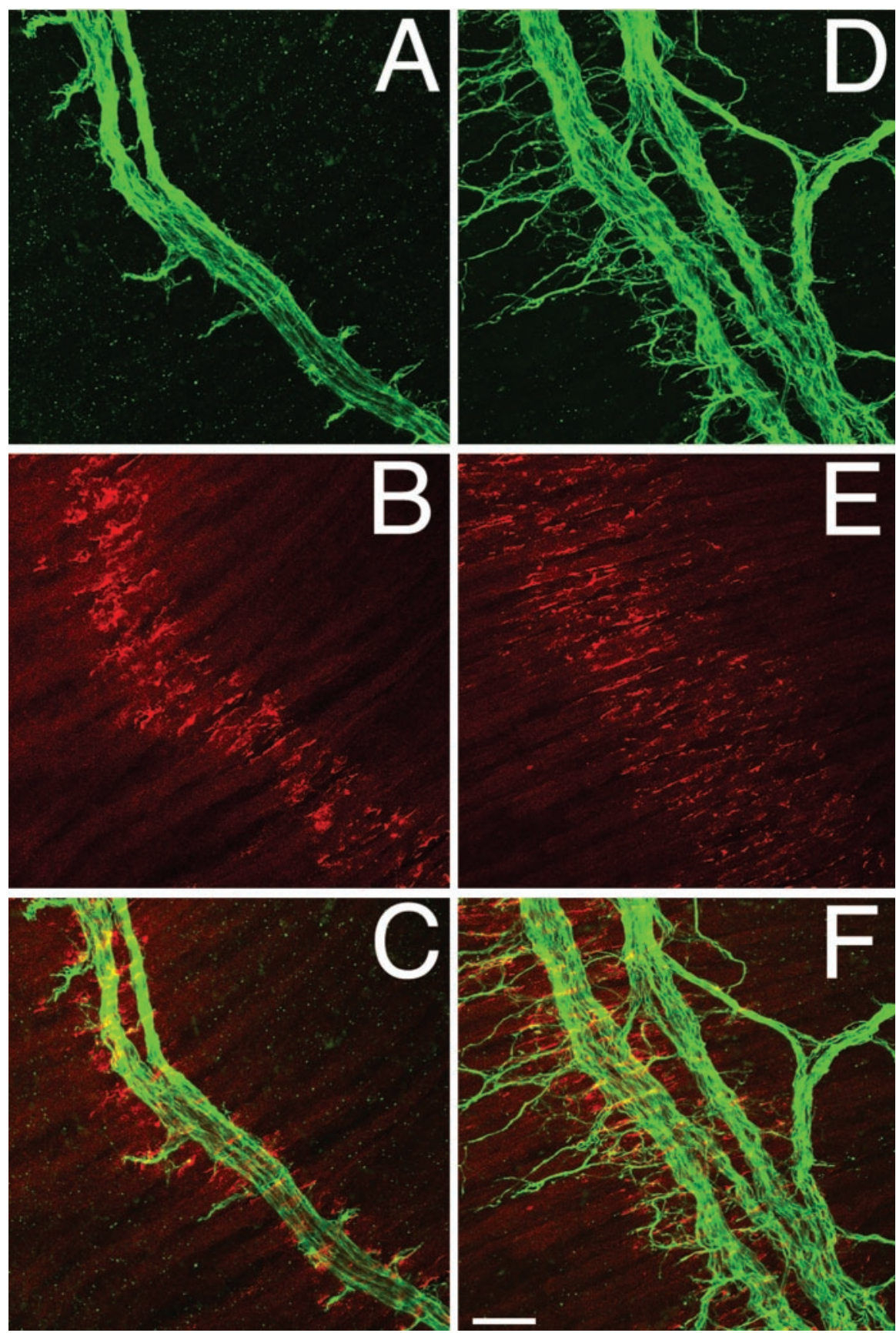

Figure 5. AChRs are clustered in the central band of Chat mutant muscle at E14.5. Whole-mount diaphragms were doublelabeled with anti-NF antibodies $(A$, control, $+/+; D$, mutant, $-/-)$ and Texas Red-conjugated $\alpha$-bungarotoxin $(B, \operatorname{control} ; E$, mutant). In both control and mutant embryos, $A C h R$ clusters are found along the central band of the muscle, although $A C h R$ clusters in Chat mutants $(E)$ are distributed over a broader region compared with those in the controls $(B)$. Merged images show that the intramuscular nerve trunk extends along the central band of $A C h R$ clusters in both control $(C)$ and Chat mutant embryos $(F)$. Note that nerves in the Chat mutants occupy a much broader region of muscle. Scale bar, $50 \mu \mathrm{m}$.

muscles were dissected out and fixed in the same solution overnight at $4^{\circ} \mathrm{C}$. The tissue was then rinsed with buffer and postfixed in $2 \%$ osmium tetroxide in buffer for $1 \mathrm{hr}$ on ice. The tissue was then dehydrated in a graded series of ethanol, infiltrated, and polymerized in Epon 812 (Polysciences, Warrington, PA). Ultrathin sections were stained with uranyl acetate and lead citrate, and electron micrographs were recorded using either a Jeol (Tokyo, Japan) 100CXII or Philips (Eindhoven, The Netherlands) EM420 electron microscope operated at $80 \mathrm{kV}$.

Electron microscopy morphometry. The electron micrographs of NMJ sections were digitized and analyzed using Scion Image software (Scion
Corporation, Frederick, MD). The following measurements were made from each presynaptic nerve terminal profile: perimeter length, nerve terminal area, synaptic contact length, active zone number, docked synaptic vesicle number, and synaptic vesicle density. Morphological criteria for distinguishing synaptic components were based on a previous study by Kelly and Zacks (1969). The synaptic contact was measured as the length of the presynaptic plasma membrane that was apposed to the postsynaptic muscle membrane at a distance of $50-80 \mathrm{~nm}$. The active zone was defined as a cluster of synaptic vesicles at the presynaptic membrane. A docked synaptic vesicle was defined as a presynaptic plasma membrane-attached vesicle at the active zone. The synaptic vesicle density was determined as the number of synaptic vesicles in a $0.04 \mu \mathrm{m}^{2}$ area surrounding the active zone or immediately adjacent to the presynaptic membrane in the region of synaptic contact for sections not passing through active zones (Herrera et al., 1985). In addition, the postsynaptic membrane length $(l)$ and the number of presynaptic nerve terminals per junction were measured. When junctional folds were present, the membrane length including the folds $(L)$ was also measured. Then, the junctional fold length was obtained by calculating $(L-l)$. A twotailed $t$ test was used to determine statistical significance.

\section{Results}

\section{Generation of ChAT-deficient mice}

To investigate the role of ACh in mammalian neural development, we established mice lacking ChAT and, hence, ACh biosynthesis. As illustrated in Figure 1, a targeting construct was generated, linearized, and electroporated into J1 ES cells (Fig. 1A). Of 843 ES cell clones screened by Southern blot analysis, two were identified that contained the disrupted Chat allele (Fig. $1 B$ ); these were injected into C57BL/ 6 blastocysts to generate chimeric mice. Germ-line transmission of the mutant allele was obtained, and the mutant allele is transmitted in a Mendelian manner (Fig. 1C). Mice heterozygous for the Chat mutation are healthy and fertile despite a marked reduction in ChAT activity (Fig. 1D). At all stages of embryonic development, one-quarter of the embryos in heterozygous cross litters were found to be homozygous Chat mutants (data not shown), indicating that no prenatal lethality is associated with the mutation. However, homozygous Chat mutant embryos die at birth, presumably because of an absence of synaptic transmission in the diaphragm muscle. That this allele represents a null mutation of the Chat gene was confirmed by an absence of ChAT activity in the brain stem, spinal cord, and septum (Fig. 1D) and of ChAT immunoreactivity in the basal forebrain (Fig. $1 E$ ) of E18.5 embryos. Morphologically, Chat mutants are shorter than control littermates (E18.5, crown-to-rump length of $19 \pm 0.2$ vs $24 \pm 0.6$ $\mathrm{mm}$, respectively; $n=7$ mutant, 4 wild type; $p<0.0001$ ) and 
display kyphosis (hunchback) and carpoptosis (wrist drop) (Fig. $1 F$ ). These overt morphological characteristics are readily apparent by approximately E15.5.

Although ChAT is believed to be the sole enzyme responsible for ACh synthesis in vivo, it is possible that the elimination of ChAT activity was not sufficient to eliminate ACh. To confirm that Chat homozygous mutant embryos were indeed deficient in ACh, we performed electrophysiological analyses of the NMJ. Intracellular recording of muscle fibers was performed in an intact nerve-muscle preparation of control and Chat mutant samples. In contrast to the control, which showed spontaneous mepps (Fig. 2A) and nerve-evoked epps (Fig. 2C), Chat mutant muscle did not show any activity (Fig. $2 A, C$ ). In addition, $40 \mathrm{~mm}$ potassium induced massive activity in control muscle but not in Chat mutant muscle (Fig. 2B). Interestingly, treatment with the ACh agonist carbachol evoked postsynaptic membrane potentials and caused muscle contraction in both mutant and control preparations (Fig. 2D), demonstrating that muscle is capable of responding to ACh, which also suggests that ACh receptors are present and functional in the Chat mutants. These electrophysiological experiments provide compelling evidence that in Chat mutants, the elimination of ChAT results in a complete elimination of neurotransmission at the NMJ.

\section{Increased innervation and spinal motor neuron survival}

To examine the innervation pattern of motor nerves in the Chat mutant mice, whole-mount diaphragm muscles from E18.5 embryos were immunostained with an anti-neurofilament (NF) antibody. As shown in Figure $3 A-D$, the phrenic nerve innervates the central band of the diaphragm muscle in the control embryos (Fig. $3 A, C$ ), but the nerve is highly branched and innervates a much broader region in the Chat mutants (Fig. 3B,D). Similar results were observed in E18.5 limb and intercostal muscles (data not shown). These results are consistent with previous studies in chick embryos treated with AChR antagonists (Burden, 1977; Pittman and Oppenheim, 1978; Srihari and Vrbova, 1978; Dahm and Landmesser, 1988, 1991; Oppenheim et al., 1989, 2000; Hory-Lee and Frank, 1995; Usiak and Landmesser, 1999). To determine whether Schwann cells are present in Chat mutants, we examined the distribution of Schwann cells in motor nerves. Immunostaining with antibody against $S 100 \beta$ revealed that Schwann cells are present and distributed along motor nerves in control (Fig. 3E) and Chat mutant embryos (Fig. 3F). Electron microscopy (EM) studies also demonstrated that terminal Schwann cells are present in mutant embryos (see Fig. 9).

Spinal motor neurons, one of the major cells that express ChAT, respond to neurotrophic factors from the target for survival. In light of increased muscle innervation, we sought to determine whether motor neuron survival is affected in ChAT mutants. Consistent with previous studies using ACh antagonists with chicks in ovo (Pittman and Oppenheim, 1978; Srihari and Vrbova, 1978; Dahm and Landmesser, 1988, 1991; Oppenheim et al., 1989, 2000; Hory-Lee and Frank, 1995; Usiak and Landmesser, 1999), homozygous Chat mutant embryos were found to have $\sim 60 \%$ more motor neurons in cervical and thoracic segments than control embryos (Fig. $3 G$ ). In addition, stereological measurement revealed that the average area of a motor neuron cross section is increased by $\sim 10 \%$ in this region $(n=7$ mutant, 5 wild type; $p<0.05$ ).

To determine when increased innervation occurs in the Chat mutant embryos, spinal cord sections from embryos at E12.5 were immunostained with an anti-NF antibody. This is the stage at which the developing phrenic nerve first arrives at the dia-


Figure 6. $\quad A C h R$ clusters are increased in number and populate a broader area of muscle in Chat mutants. E18.5 diaphragm muscles from control $(A)$ and mutant $(B)$ embryos were labeled with Texas Red-conjugated $\alpha$-bungarotoxin. AChRs are clustered along a central band of muscle in both the control $(A)$ and the mutant $(B)$, although there are more AChR clusters in the mutant, and they occupy a broader region (compare $B$ with $A$ ). $C$, Histogram illustrating the distribution of $A C h R$ clusters. Numbers of $A C h R$ clusters in the right ventral-costal portion of diaphragm muscles (within an area of $4.8 \times 10^{5} \mu \mathrm{m}^{2}$, as shown in $A$ and $B$ ) from E18.5 control $(n=3)$ and mutant $(n=3)$ embryos were counted. The $x$-axis is the distance to the medial edge of the muscle fibers (in $0.1 \mathrm{~mm}$ intervals); the $y$-axis indicates the average number of $A C h R$ clusters in each $0.1 \mathrm{~mm}$ interval. Scale bar, $100 \mu \mathrm{m}$.

phragm muscle and is before the clustering of muscle AChRs (Lupa and Hall, 1989; Lin et al., 2000). Our results showed that motor nerves exit the spinal cord normally and remain fasciculated en route to their muscle target in the Chat mutant embryos (data not shown). However, on contact with the diaphragm muscle, a marked increase in nerve branching is observed in the Chat mutant embryos (Fig. 4B), whereas the phrenic nerve remains bundled, with limited and defined growth in the control embryos (Fig. 4A). Similar results were observed in E12.5 intercostal muscles (Fig. $4 C, D$ ). Increased innervation becomes more dramatic as embryos develop to later stages (E13.5: Fig. 4E,F; E18.5: Fig. $3 A, B)$. These results demonstrate that the muscle is hyperinnervated by motor nerves on initial contact and before the onset of AChR clustering.

\section{Aberrant patterning of neuromuscular synapses}

We subsequently analyzed the pattern of developing neuromuscular synapses (E14.5-E18.5) in Chat mutants. To examine the distribution of nerves and AChR clusters at E14.5, the earliest stages at which clustering of AChRs becomes detectable (Lupa and Hall, 1989; Lin et al., 2001), diaphragm muscle was labeled with anti-NF antibody and $\alpha$-bungarotoxin. As shown in Figure 5, AChRs are clustered within a 




Figure 7. Aberrant patterning of neuromuscular synapses in Chat mutants. Diaphragm muscles were collected from E18.5 control $(A, C-E ;+/+)$ and mutant $(B, F-H ;-/-)$ embryos and immunostained with an anti-synaptophysin antibody ( green) and Texas Red-conjugated $\alpha$-bungarotoxin (red). Synapses are more broadly distributed across the muscle in mutant $(B)$ compared with control $(A)$ embryos, especially in the dorsal portions of the diaphragm, the pars costalis and crus laterale. Synaptophysin-positive nerve terminals $(C, F)$ and $A C h R$ clusters $(D, G)$ occupy a much broader territory in the mutants compared with the control littermates. Merged confocal images show that all $A C h R$ clusters are colocalized with nerve terminals $(E, H)$. Scale bars: (in $B) A, B, 500 \mu \mathrm{m}$; (in $H) C-H, 50 \mu \mathrm{m}$.

central band of the muscle in both control (Fig. 5B) and mutant (Fig. $5 E$ ) embryos, but the band of AChR clusters is much broader than normal in the mutant. Interestingly, intramuscular nerves in the mutant also occupy a broader region (Fig. $5 D, F$ ) than those in control embryos (Fig. 5A,C).

As development proceeds to later stages, the AChR clustering band remains broadened in Chat mutants. Figure 6 shows the distribution and quantification of AChR clusters in E18.5 diaphragm muscles. Because the distribution pattern varies along the dorsoventral axis, anatomically matched areas of control and mutant diaphragm muscles were analyzed. We quantified AChR clusters in a $4.8 \times 10^{5} \mu \mathrm{m}^{2}$ area of the right ventral-costal portion of each diaphragm (Fig. $6 A, B$ ). Within this area, the number of AChR clusters is significantly increased in the mutants (284 \pm 12 , controls; $467 \pm 18$, mutants; $p<0.001)$. As shown in Figure $6 C, A C h R$ clusters are distributed across a broader area in the mutants compared with those in the control embryos. The end plate band in Chat mutants is approximately three times the width of that in controls. That is, in the controls, $90 \%$ of the AChR clusters are distributed within a central band $200 \mu \mathrm{m}$ in width, whereas in the mutants, a $600-\mu \mathrm{m}$-wide band must be delineated to encompass $90 \%$ of the AChR clusters. Only $47 \%$ of the total clusters are found in the central $200 \mu \mathrm{m}$ band in the mutants. These results demonstrate that AChR clusters are increased in number and distributed in a broader region of the diaphragm muscle in the Chat mutants.

To determine the spatial relationship between nerve terminals and AChR clusters, whole-mount diaphragm muscles (E18.5) were immunostained with anti-synaptophysin and $\alpha$-bungarotoxin. As shown in Figure 7, synaptophysin-immunoreactive nerve terminals are broadly distributed in E18.5 mutants (Fig. 7B) compared with the delimitation of terminals to the central band observed in control embryos (Fig. $7 A$ ). High-power images show that all AChR clusters are directly apposed by nerve terminals in both control (Fig. 7E) and mutant (Fig. $7 H$ ) embryos.

In addition to AChR clustering, we examined the distribution of AChE in Chat mutant embryos. As shown in Figure $9 A, B, A C h E$ clusters are also distributed in a broader region of muscle in Chat mutant embryos compared with control embryos, consistent with previous results from chick embryos treated with blockers of cholinergic neurotransmission (Gordon and Vrbova, 1975).

\section{Ultrastructure of neuromuscular synapses is impaired in \\ mutant embryos}

We subsequently determined whether $\mathrm{ACh}$ is required for synapse formation as examined at the ultrastructural level. EM observation of E17.5 diaphragm muscles revealed that the major synaptic features found in the control NMJ were also found in the Chat mutant NMJ. However, morphometric analysis detected significant defects in the mutant NMJs, including smaller nerve terminals, fewer synaptic contacts, and fewer junctional folds than in control NMJs.

As shown in Figure 8, typical features of embryonic NMJs were observed in both mutants (Fig. $8 \mathrm{~A}$ ) and controls (Fig. 8D). At this stage of development, NMJs in both genotypes have multiple motor nerve terminals (Fig. 8, N), which make synaptic contacts on the postsynaptic membrane of muscle fiber (Fig. 8, $M)$, with few junctional folds. The neuromuscular contact areas are capped by perisynaptic Schwann cells (Fig. 8, S), whose processes are closely associated with the nerve terminals. The basal lamina (Fig. 8, arrowheads) is found in the synaptic cleft in both genotypes. The nerve terminals in both mutant and control NMJs contain clusters of synaptic vesicles. At the presynaptic membrane, active zones consisting of a cluster of synaptic vesicles including docked vesicles are occasionally seen in both genotypes (Fig. 8B,F). However, as shown in Figure $8 C$, in some mutant NMJs, the nerve terminals are smaller and make fewer synaptic contacts. Electron-lucent areas (Fig. 8C, asterisks) in the synaptic intercellular space are more prevalent in mutant NMJs than in control NMJs. Another striking difference is in the postsynaptic 
membrane. As shown in Figure $8 E$, well developed junctional folds were found in approximately one-third of control NMJs (5 of 16 NMJs). In contrast, no such junctional folds were observed in any of the 13 mutant NMJs examined.

To further investigate quantitative differences between mutant and control NMJs, we compared various parameters of the presynaptic nerve terminals. As summarized in Table 1, the area and perimeter of the nerve terminals and the synaptic contact length in mutant NMJs are significantly smaller than those in controls. The nerve terminals in mutant and control NMJs contain similar numbers of active zones and docked synaptic vesicles despite the slightly lower synaptic vesicle density in the mutant. The number of nerve terminals per NMJ and the postsynaptic membrane length are not different between mutants and controls. Thus, at the ultrastructural level, several features, including both presynaptic and postsynaptic components, appear to be altered in Chat mutant NMJs.

\section{AChR transcripts are concentrated at synaptic sites in the Chat mutants}

We subsequently examined the expression of the AChR by in situ hybridization using probes specific to the $\alpha$-subunit gene of AChR. We first analyzed the whole-mount preparation of the diaphragm muscle. Whole-mount in situ hybridization shows that AChR transcripts are confined to the middle of muscle in the control and mutant (Fig. 9C,D), although the in situ signal appears in a broader region in the mutants (Fig. 9D). Interestingly, the $\mathrm{AChR}$ transcript is found in a pattern similar to that of AChE staining in both genotypes (Fig. 9, compare $A$ with $C$ and $B$ with $D$ ). Because AChE clusters are reliable markers of synaptic sites (Moscoso et al., 1995; Schaeffer et al., 1998), these results suggest that the AChR $\alpha$-subunit gene is transcribed only at synaptic sites. We also investigated this by double-labeling the muscle with AChE staining and in situ hybridization with ${ }^{33} \mathrm{P}$-labeled AChR $\alpha$ riboprobe. As shown in Figure 9E-J, AChE and AChR $\alpha$ transcript clusters are colocalized in both control (Fig. 9E,F) and mutant (Fig. 9G,H) embryos. High-power bright-field micrographs show that the silver grains for $\mathrm{AChR} \alpha$ mRNA are superimposed on the AChE stain in both control (Fig. 9I) and mutant (Fig. 9J) embryos. No signal above background levels was detected outside of AChE clusters in either control or mutant embryos. Thus, the combined
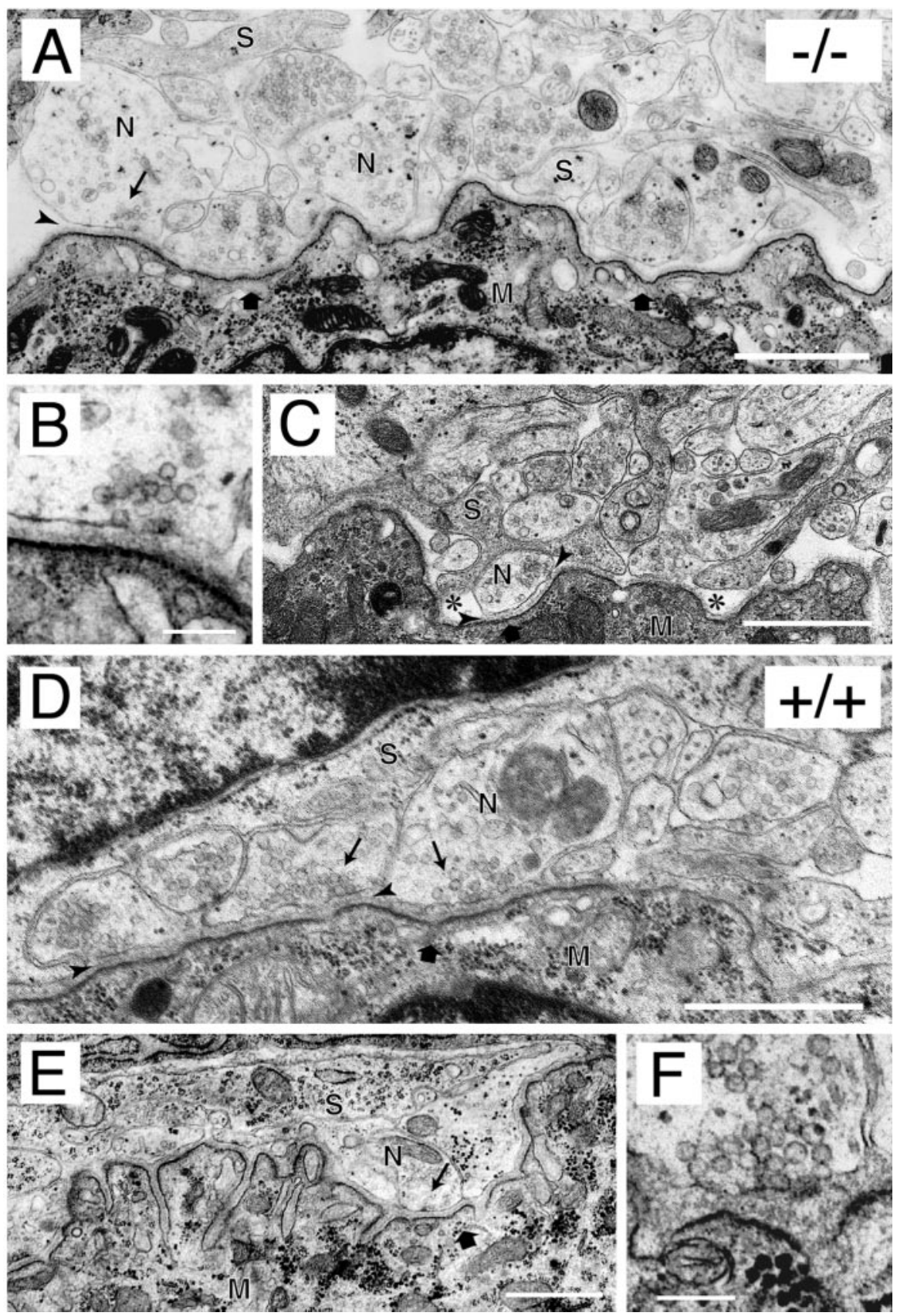

Figure 8. Ultrastructure of the NMJ in Chat mutant mice. Electron micrographs of NMJs from E17.5 Chat null mutant (A-C; $-/-)$ and control $(D-F ;+I+)$ diaphragm muscles are shown. $A$, A representative micrograph from a Chat mutant shows features typical of embryonic NMJs. The multiple motor nerve terminals $(N)$, capped by the processes of perisynaptic $S c h w a n n$ cells $(S)$, make synaptic contacts on the postsynaptic membrane of the muscle cells $(M)$. In mutants, the postsynaptic membrane has only indentations (large arrows) and lacks junctional folds. The basal lamina (arrowheads) is seen in the synaptic cleft. The nerve terminals contain mitochondria and clusters of synaptic vesicles (arrow). $B$, A higher magnification of the area in $A$ indicated by the arrow depicts an active zone with a docked synaptic vesicle in the mutant nerve terminal. C, A representative NMJ that illustrates some of the alterations observed in Chat mutants. This NMJ appears to have smaller nerve terminals and makes fewer synaptic contacts than normal, although all of the synaptic components described above are present. Note the prevalent electron-lucent areas (asterisks) in the synaptic cleft. D, A representative control NMJ also shows features typical of the embryonic NMJ, including the multiple nerve terminals $(N)$, the perisynaptic Schwann cell $(S)$, and the basal lamina (arrowheads). Only slight indentations (large arrow) are observed in the postsynaptic membrane. The clusters of synaptic vesicles (arrows) are clearly seen in the nerve terminals. $E$, An example of a well developed NMJ from control embryos shows large indentations of the postsynaptic membrane (large arrow) and elaborate junctional folds. This more mature feature, found in one-third of NMJs from control embryos, was never found in mutant embryos. $F$, Higher magnification of an adjacent section in $E$ indicated by the arrow depicts a cluster of synaptic vesicles over a junctional fold, resembling the active zone in mature NMJs. Scale bars: $A, C-E, 1 \mu \mathrm{m} ; B, F, 0.2 \mu \mathrm{m}$. 
Table 1. Comparison of ultrastructural parameters in presynaptic motor nerve terminals from Chat mutant and control embryos

\begin{tabular}{lcc}
\hline & Mutant & Control \\
\hline Nerve terminal parameters & $n=55$ & $n=54$ \\
Nerve terminal area $\left(\mu \mathrm{m}^{2}\right)$ & $0.36 \pm 0.05^{* *}$ & $0.66 \pm 0.09$ \\
Nerve terminal perimeter $(\mu \mathrm{m})$ & $2.38 \pm 0.17^{* * *}$ & $3.37 \pm 0.24$ \\
Synaptic contact length $(\mu \mathrm{m})$ & $0.58 \pm 0.08^{* *}$ & $1.07 \pm 0.12$ \\
Synaptic vesicle density $\left(\right.$ per $\left.0.04 \mu \mathrm{m}^{2}\right)$ & $4.11 \pm 0.30^{*}$ & $5.00 \pm 0.30$ \\
Active zone number & $0.24 \pm 0.06$ & $0.33 \pm 0.08$ \\
Docked synaptic vesicle number & $0.45 \pm 0.09$ & $0.58 \pm 0.10$ \\
NMJ parameters & $n=13$ & $n=16$ \\
Nerve terminal number/NMJ & $4.23 \pm 0.76$ & $3.38 \pm 0.42$ \\
Postsynaptic membrane length/NMJ $(\mu \mathrm{m})$ & $6.00 \pm 0.61$ & $6.99 \pm 0.73$ \\
Total postsynaptic membrane length $(\mu \mathrm{m})$ & 78.05 & 111.83 \\
Total junctional fold length $(\mu \mathrm{m})$ & 0.55 & 21.82 \\
\hline
\end{tabular}

Morphometric analysis was performed on EM images of NMJs from E17.5 diaphragm muscles. The definition of parameters is described in Materials and Methods. Data were collected from 55 nerve terminals of 13 NMJs in three mutants and 54 nerve terminals of $16 \mathrm{NMJs}$ in three control embryos and are expressed as means \pm SEM. Statistical significance was assessed by two-tailed $t$ test; ${ }^{*} p<0.05 ;{ }^{* *} p<0.01$; ${ }^{* * *} p<0.001$.

AChE staining and radioactive in situ hybridization confirm that $\mathrm{AChR} \alpha$ transcripts are concentrated at synaptic sites in Chat mutants, although, like the synapses, the overall distribution pattern of AChR $\alpha$ transcripts is broader than normal.

\section{Discussion}

In the present study, we analyzed neuromuscular development in Chat null mutant mice. In the Chat mutant NMJ, both spontaneous and nerve-evoked postsynaptic potentials are absent. Motor nerves elaborate supernumerary axon bundles on contact with the muscle at E12.5 and continue to grow and innervate a broader region of muscle at E14.5, including the central band, where AChR clusters are present. At E16.5-E18.5, nerve terminals become differentiated and release other signals to induce synapse formation. Likely as a result of the increased innervation,

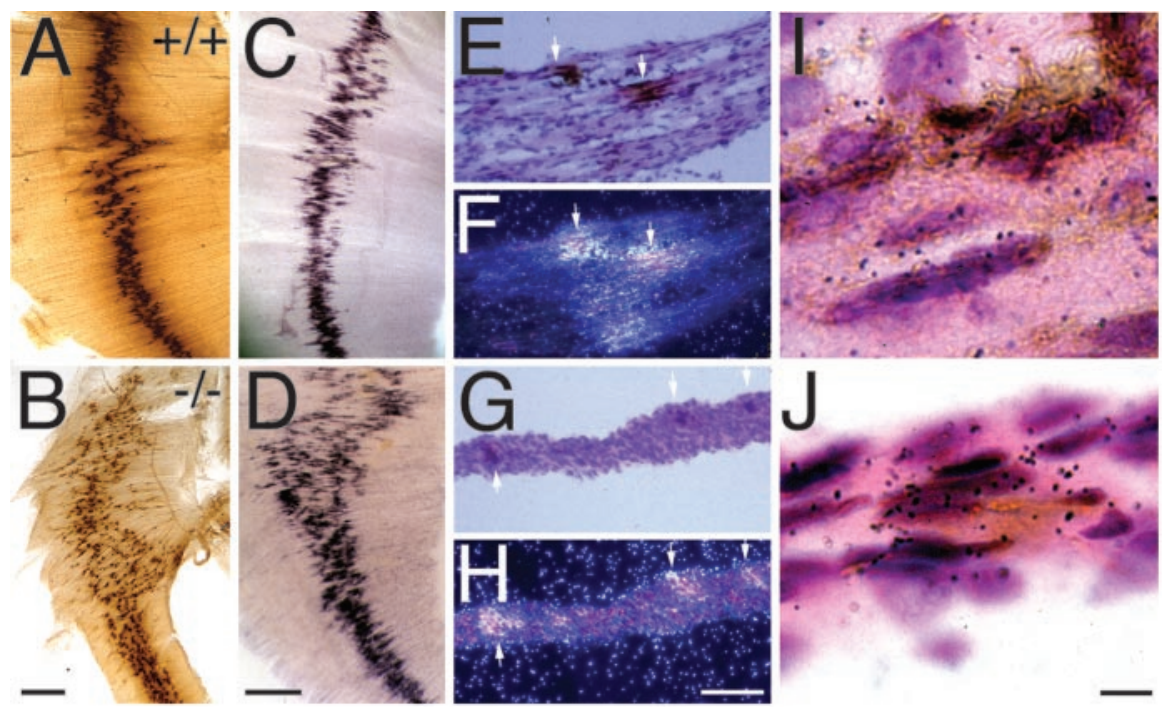

Figure 9. Distribution of $A C h E$ clusters and $A C h R$ mRNA in the Chat mutants. Diaphragm muscles were collected from E17.5 control $(A, C, E, F, I ;+/+)$ and mutant $(B, D, G, H, J ;-/-)$ embryos and subjected to $A C h E$ histochemistry $(A, B)$, whole-mount in situ hybridization $(C, D)$, or combined $A C h E /$ radioactive in situ hybridization $(E-J)$. It is apparent that by late gestation, muscle is less well developed in mutant compared with wild-type embryos. In mutant embryos, $A C h E(B)$ was clustered in a pattern similar to that of nerve terminals (Fig. 7B). Whole-mount in situ hybridization revealed that AChR $\alpha$ mRNA was concentrated $(D)$ in a pattern similar to that of $A C h E$ clusters $(B)$ in the mutants. AChE clusters $(E, G)$ and $A C h R \alpha$ transcripts (dark field; $F, H$ ) were colocalized (arrows) in both control $(E, F)$ and mutant $(G, H)$ diaphragm transverse sections. I, J, High-power bright-field micrographs from $E$ and $G$. The results show that silver grains coincide with $A C h E$ aggregates both in control $(I)$ and in mutant $(J)$ embryos. Scale bars: (in B, D) A-D, $500 \mu \mathrm{m}$; (in H) E-H, $200 \mu \mathrm{m}$; (in J) I-J, $15 \mu \mathrm{m}$. synapses in mutants are distributed in a much broader region of muscle than in control embryos. Although AChRs are clustered in the Chat mutants, the fine structure of the mutant synapses is altered (Table 1). Interestingly, Verhage et al. (2000) have recently generated mice lacking the synaptic vesicle protein mammalian homolog of unc-18 (Munc18-1), in which no synaptic transmission is detected at the NMJ. In the Munc18-1 mutant embryos, AChRs are clustered as well, although the ultrastructure of their synapses has not been reported. Our findings of increased muscle innervation and motor neuron survival are also consistent with previous studies using cholinergic or neuromuscular activity blockers in chick embryos (Pittman and Oppenheim, 1978; Dahm and Landmesser, 1988, 1991; Oppenheim et al., 1989, 2000; Hory-Lee and Frank, 1995; Usiak and Landmesser, 1999).

Because ChAT is a critical enzyme for the biosynthesis of ACh, the phenotypic characteristics of the Chat mutant embryos are likely attributable to the loss of ACh, although we cannot rule out the possibility that ChAT has other nonenzymatic activities that might contribute to these changes. In addition to the role of ACh in ionotropic activity, it is possible that ACh plays a role in activities independent of synaptic transmission. Also, although the critical source of ACh is most likely neuronal, Schwann cells have been shown to express ChAT in culture (Brockes, 1984), so it is possible that Schwann cell-derived ACh has a role in the patterning of neuromuscular synapses.

Neuromuscular alterations in the Chat mutants might result from defects in ACh-dependent activities at presynaptic and/or postsynaptic sites. Relevant to this possibility, it is known that different AChR subtypes are present at nerve terminals, perisynaptic Schwann cells, and muscle fibers. Neuronal nicotinic AChRs, distinct from those in the muscle, are expressed at preterminals and presynaptic terminals (Wessler, 1992; Role and Berg, 1996), whereas muscarinic AChR subtypes are detected in perisynaptic Schwann cells (Rochon et al., 2001). Thus, different cholinergic receptors may mediate each of these processes. Because altered innervation is observed in mutants as early as E12.5, a stage at which AChRs are diffusely distributed in the muscle (Bevan and Steinbach, 1977), the regulation of nerve growth, at least at early stages, is probably a presynaptic process. ACh is released from growing nerve processes and terminals before their contact with muscle (Hume et al., 1983; Young and Poo, 1983; Zakharenko et al., 1999) and acts through preterminal AChRs to regulate fasciculation and nerve branching and to affect growth cone turning in vitro (Zheng et al., 1994) in a $\mathrm{Ca}^{2+}$-dependent manner (Hong et al., 2000). Alternatively, or in addition, initial cholinergic activity at early E12.5 may evoke a retrograde signal from the muscle and/or Schwann cells to the nerve terminal that inhibits overgrowth, limits branching of nerve terminals, or promotes precise target recognition (Fitzsimonds and Poo, 1998). Finally, the increased survival of motor neurons may result in more nerve being present in the muscle, and consequently, innervation of a larger area of muscle. That is, in the Chat mutants, simply the overabun- 
dance of axons may force some into territory that they normally would not innervate.

Consistent with the idea that ChAT/ACh plays a role in restricting the development of nerve terminals to the central band of muscle, Patel and Poo (1984) demonstrated that growth cones can be guided to, and will stop growing at, a focal electrical field. When the focal electrical field is turned off, growth cones resume migration away from the focal field. Because nerve-independent AChR clusters are formed in the central band of muscle at E14.5, ACh may act through these AChR clusters to establish a focal electrical field, and in this way could cause nerve terminals to arrest their growth at the central band of muscle. In addition, such activation may render the region outside of the central band nonreceptive for nerve terminals. Although the central band of muscle appears to contain an intrinsic signal to initiate AChR clustering (Lin et al., 2001; Yang et al., 2001), our results reveal that, although they do not normally use it, muscle regions outside of the central band do indeed contain the machinery necessary for synapse formation as well. In accordance with these results, we suggest that ACh plays a role in preventing nerve terminals from growing into the region outside of the central band of muscle.

Increased motor neuron survival in Chat mutant embryos may be attributable to alterations in one or more of four different cellular activities. First, an absence of synaptic transmission may lead to elevated production of neurotrophic factors in the Chat mutant embryos. However, previous studies have demonstrated that neither muscle denervation nor inactivity induced by neurotoxins results in the increased neurotrophic factor levels available for chick motor neuron survival (Houenou et al., 1991). Second, the increased muscle innervation may increase the access of motor neurons to neurotrophic factors for survival. Consistent with this idea, motor neuron number is increased in several mutant mice that exhibit increased muscle innervation, including agrin and muscle-specific kinase mutants (Terrado et al., 2001). In contrast, loss of muscle innervation is correlated with increased cell death, as shown in erbB2 and erbB3 mutant mice (Riethmacher et al., 1997; Morris et al., 1999). Third, because motor neurons also express AChRs, ACh may directly regulate motor neuron survival via a central mechanism (Hory-Lee and Frank, 1995). However, recent results obtained by Oppenheim et al. (2000) do not support this idea. They found that neuromuscular activity blockers, but not neuronal nicotinic AChR blockers alone, increase chick motor neuron survival. Finally, the lack of ACh signaling through these receptors may make motor neurons more responsive to muscle-derived neurotrophic factors. Consistent with this last possibility, it has been shown that nicotinic blocking agents, such as dTC, potentiate the ability of muscle extracts to effect motor neuron survival in culture (Hory-Lee and Frank, 1995).

Finally, is our finding regarding the regulation of synaptogenesis by neurotransmitter unique to ACh in mammals? Remarkably, elimination of ACh in Drosophila leads to increased branching of retinal axons (S. Kunes, personal communication). Other neurotransmitters also appear to be important for synaptic development. Glutamate has been shown to regulate the synaptic location of glutamate receptor clusters in rat hippocampal neurons (Rao and Craig, 1997) and of the postsynaptic receptor fields of the Drosophila NMJ (Featherstone et al., 2000). Similarly, GABA has been shown to regulate GABAergic synaptogenesis, including the expression of a GABA receptor subtype (Belhage et al., 1998) and the developmental switch of synaptic responses from excitation to inhibition (Ganguly et al., 2001). In summary, our results are consistent with the emerging theme that neurotransmitters actively regulate the development of the neurons and the synapses they subserve.

\section{References}

Belhage B, Hansen G, Elster L, Schousboe A (1998) Effects of $\gamma$-aminobutyric acid (GABA) on synaptogenesis and synaptic function. Perspect Dev Neurobiol 5:235-246.

Bevan S, Steinbach JH (1977) The distribution of $\alpha$-bungarotoxin binding sites on mammalian skeletal muscle developing in vivo. J Physiol (Lond) 267:195-213.

Braithwaite AW, Harris AJ (1979) Neural influence on acetylcholine receptor clusters in embryonic development of skeletal muscles. Nature 279:549-551.

Brandon EP, Lin W, D’Amour KA, Pizzo DP, Dominguez B, Thode S, Thal LJ, Lee KF, Gage FH (2000) Choline acetyltransferase knockout mice have defects in neuromuscular development. Soc Neurosci Abstr 26:411.8.

Brockes JP (1984) Assays for cholinergic properties in cultured rat Schwann cells. Proc R Soc Lond B Biol Sci 222:121-134.

Burden S (1977) Development of the neuromuscular junction in the chick embryo: the number, distribution, and stability of acetylcholine receptors. Dev Biol 57:317-329.

Burden SJ (1998) The formation of neuromuscular synapses. Genes Dev 12:133-148.

Carbini LA, Hersh LB (1993) Functional analysis of conserved histidines in choline acetyltransferase by site-directed mutagenesis. J Neurochem 61:247-253.

Dahm LM, Landmesser LT (1988) The regulation of intramuscular nerve branching during normal development and following activity blockade. Dev Biol 130:621-644.

Dahm LM, Landmesser LT (1991) The regulation of synaptogenesis during normal development and following activity blockade. J Neurosci 11:238-255.

Davis GW, Eaton B, Paradis S (2001) Synapse formation revisited. Nat Neurosci 4:558-560.

Featherstone DE, Rushton EM, Hilderbrand-Chae M, Phillips AM, Jackson FR, Broadie K (2000) Presynaptic glutamic acid decarboxylase is required for induction of the postsynaptic receptor field at a glutamatergic synapse. Neuron 27:71-84.

Ferns M, Carbonetto S (2001) Challenging the neurocentric view of neuromuscular synapse formation. Neuron 30:311-314.

Fitzsimonds RM, Poo M-M (1998) Retrograde signaling in the development and modification of synapses. Physiol Rev 78:143-170.

Ganguly K, Schinder A, Wong S, Poo M-M (2001) GABA itself promotes the developmental switch of neuronal GABAergic responses from excitation to inhibition. Cell 105:521-532.

Gordon T, Vrbova G (1975) Changes in chemosensitivity of developing chick muscle fibres in relation to endplate formation. Pflügers Arch 360:349-364.

Hahn M, Hahn SL, Stone DM, Joh T (1992) Cloning of the rat gene encoding choline acetyltransferase, a cholinergic neurons-specific marker. Proc Natl Acad Sci USA 89:4387-4391.

Herrera AA, Grinnell AD, Wolowske B (1985) Ultrastructural correlates of naturally occurring differences in transmitter release efficacy in frog motor nerve terminals. J Neurocytol 14:193-202.

Hong K, Nishiyama M, Henley J, Tessier-Lavigne M, Poo M-M (2000) Calcium signalling in the guidance of nerve growth by netrin-1. Nature 403:93-98.

Hory-Lee F, Frank E (1995) The nicotinic blocking agents $d$-tubocurare and $\alpha$-bungarotoxin save motoneurons from naturally occurring death in the absence of neuromuscular blockade. J Neurosci 15:6453-6560.

Houenou LJ, Pincon-Raymond M, Garcia L, Harris AJ, Rieger F (1990) Neuromuscular development following tetrodotoxin-induced inactivity in mouse embryos. J Neurobiol 21:1249-1261.

Houenou LJ, McManaman JL, Prevette D, Oppenheim RW (1991) Regulation of putative muscle-derived neurotrophic factors by muscle activity and innervation: in vivo and in vitro studies. J Neurosci 11:2829-2837.

Hume RI, Role LW, Fischbach GD (1983) Acetylcholine release from growth cones detected with patches of acetylcholine receptor-rich membranes. Nature 305:632-637.

Karczmar AG (1996) The Otto Loewi lecture: Loewi's discovery and the XXI century. Prog Brain Res 109:1-27. 
Kelly AM, Zacks SI (1969) The fine structure of motor endplate morphogenesis. J Cell Biol 42:154-169.

Lee K-F, Li E, Huber LJ, Landis SC, Sharpe AH, Chao MC, Jaenisch R (1992) Targeted mutation of the gene encoding the low affinity NGF receptor p75 leads to deficits in the peripheral sensory nervous system. Cell 69:737-749.

Lin W, Sanchez HB, Deerinck T, Morris JK, Ellisman M, Lee K-F (2000) Aberrant development of motor axons and synapses in erbB2-deficient mice. Proc Natl Acad Sci USA 97:1299-1304.

Lin W, Burgess RW, Dominguez B, Pfaff SL, Sanes JR, Lee K-F (2001) Distinct roles of nerve and muscle in postsynaptic differentiation of the neuromuscular synapse. Nature 410:1057-1064.

Lupa MT, Hall ZW (1989) Progressive restriction of synaptic vesicle protein to the nerve terminal during development of the neuromuscular junction. J Neurosci 9:3937-3945.

Morris JK, Lin W, Hauser C, Marchuk Y, Getman D, Lee K-F (1999) Genetic rescue of cardiac defect in erbB2 null mutant mice reveals essential roles of erbB2 in development of the peripheral nervous system. Neuron 23:273-283.

Moscoso LM, Merlie JP, Sanes JR (1995) N-CAM, 43K-rapsyn, and $\mathrm{S}$-laminin mRNAs are concentrated at synaptic sites in muscle fibers. Mol Cell Neurosci 6:80-89.

Ohno K, Tsujino A, Brengman JM, Harper CM, Bajzer Z, Udd B, Beyring R, Robb S, Kirkham FJ, Engel AG (2001) Choline acetyltransferase mutations cause myasthenic syndrome associated with episodic apnea in humans. Proc Natl Acad Sci USA 98:2017-2022.

Oppenheim RW, Bursztajn S, Prevette D (1989) Cell death of motoneurons in the chick embryo spinal cord. XI. Acetylcholine receptors and synaptogenesis in skeletal muscle following the reduction of motoneuron death by neuromuscular blockade. Development 107:331-341.

Oppenheim RW, Prevette D, D'Costa A, Wang S, Houenou LJ, McIntosh JM (2000) Reduction of neuromuscular activity is required for the rescue of motoneurons from naturally occurring cell death by nicotinic-blocking agents. J Neurosci 20:6117-6124.

Patel NB, Poo M-M (1984) Perturbation of the direction of neurite growth by pulsed and focal electric fields. J Neurosci 4:2939-2947.

Pittman RH, Oppenheim RW (1978) Neuromuscular blockade increases motoneurone survival during normal cell death in the chick embryo. Nature 271:364-366.

Pizzo DP, Waite JJ, Thal LJ, Winkler J (1999) Intraparenchymal infusions of 192 IgG-saporin: development of a method for selective and discrete lesioning of cholinergic basal forebrain nuclei. J Neurosci 91:9-19.
Rao A, Craig AM (1997) Activity regulates the synaptic localization of the NMDA receptor in hippocampal neurons. Neuron 19:801-812.

Riethmacher D, Sonnenberg-Riethmacher E, Brinkmann V, Yamaai T, Lewin GR, Birchmeier C (1997) Severe neuropathies in mice with targeted mutations in the ErbB3 receptor. Nature 389:725-730.

Rochon D, Rousse I, Robitaille R (2001) Synapse-glia interactions at the mammalian neuromuscular junction. J Neurosci 21:3819-3829.

Role LW, Berg DK (1996) Nicotinic receptors in the development and modulation of CNS synapses. Neuron 16:1077-1085.

Sanes JR, Lichtman JW (1999) Development of the vertebrate neuromuscular junction. Annu Rev Neurosci 22:389-442.

Schaeffer L, Duclert N, Huchet-Dymanus M, Changeux J-P (1998) Implication of a multisubunit Ets-related transcription factor in synaptic expression of the nicotinic acetylcholine receptor. EMBO J 17:3078-3090.

Srihari T, Vrbova G (1978) The role of muscle activity in the differentiation of neuromuscular junctions in slow and fast chick muscles. J Neurocytol 7:529-540.

Terrado J, Burgess RW, DeChiara T, Yancopoulos G, Sanes JR, Kato AC (2001) Motoneuron survival is enhanced in the absence of neuromuscular junction formation in embryos. J Neurosci 21:3144-3150.

Usiak MF, Landmesser LT (1999) Neuromuscular activity blockade induced by muscimol and $d$-tubocurarine differentially affects the survival of embryonic chick motoneurons. J Neurosci 19:7925-7939.

Verhage M, Maia AS, Plomp JJ, Brussaard AB, Heeroma JH, Vermeer H, Toonen RF, Hammer RE, van den Berg T, Missler M, Geuze HJ, Südhof TC (2000) Synaptic assembly of the brain in the absence of neurotransmitter secretion. Science 287:864-869.

Wessler I (1992) Acetylcholine at motor nerves: storage, release, and presynaptic modulation by autoreceptors and adrenoceptors. Int Rev Neurobiol 34:283-384.

Yang X, Arber S, William C, Li L, Tanabe Y, Jessell TM, Birchmeier C, Burden SJ (2001) Patterning of muscle acetylcholine receptor gene expression in the absence of motor innervation. Neuron 30:399-410.

Young SH, Poo M-M (1983) Spontaneous release of transmitter from growth cones of embryonic neurones. Nature 305:634-637.

Zakharenko S, Chang S, O’Donoghue M, Popov S (1999) Neurotransmitter secretion along growing nerve processes: comparison with synaptic vesicle exocytosis. J Cell Biol 144:507-518.

Zheng JQ, Felder M, Connor JA, Poo M-M (1994) Turning of nerve growth cones induced by neurotransmitters. Nature 368:140-144. 